\title{
Hierarchical optimisation strategy for energy scheduling and volt/var control in autonomous clusters of microgrids
}

ISSN 1752-1416

Received on 29th March 2019

Revised 16th May 2019

Accepted on 29th May 2019

doi: 10.1049/iet-rpg.2019.0357

www.ietdl.org

\author{
Manuel V. Castro ${ }^{1}$, Carlos Moreira ${ }^{1,2}$, Leonel M. Carvalho ${ }^{1}$ \\ ${ }^{1}$ Centre for Power and Energy Systems (CPES), Institute for Systems and Computer Engineering, Technology and Science (INESC TEC), Rua \\ Dr. Roberto Frias, Porto, Portugal \\ ${ }^{2}$ Department of Electrical and Computer Engineering, Faculty of Engineering of the University of Porto (FEUP), Rua Dr. Roberto Frias, Porto, \\ Portugal \\ 凶E-mail: manuel.v.castro@inesctec.pt
}

\begin{abstract}
This study proposes a hierarchical optimisation strategy for the energy dispatch and volt/var control problem of a photovoltaic-battery microgrid cluster (MGC) operating autonomously. The proposed approach takes advantage of the decentralised control architecture existing in multi-microgrids (MMGs) framework by distributing the management responsibilities between the microgrid central controllers (MGCCs) and the central autonomous management controller (CAMC). In the first stage, the optimisation strategy solves a multi-temporal active power scheduling problem for the MGC based on consumption and generation forecasts. In the second stage, the reactive power and volt/var control are addressed by taking into account the medium-voltage (MV) and low-voltage levels independently. For this purpose, each MGCC computes the $V(Q)$ capability area of operation at the boundary bus with the MV grid. Then, the CAMC performs an optimal power flow at the MV level for each time step, whose results at the boundary bus are considered in the last stage to schedule reactive power at the MGCC level. The effectiveness of the proposed strategy is demonstrated in a cluster of three microgrids. It keeps the modularity, interoperability and scalability characteristics of the MMG concept by clearly defining the roles and the information to be exchanged between the CAMC and the MGCC.
\end{abstract}

\section{Nomenclature}

$B_{i k} \quad$ susceptance from bus $i$ to bus $k$

$G_{i k} \quad$ conductance from bus $i$ to bus $k$

$H$ total number of hours of expected autonomous operation

$K$ total number of photovoltaic (PV) generators in the microgrid cluster (MGC)

$N_{\mathrm{b}}, N_{\mathrm{s}} \quad$ total number of buses/energy storage devices

$P \quad$ active power

$P_{\mathrm{Cur}_{i}}^{h} \quad$ active power curtailed from PV unit $i$ at hour $h$

$P_{C_{i}}^{h}, P_{D_{i}}^{h}$ active power provided from charging/discharging the storage device (VSI) $i$ at hour $h$

$P_{G}^{h} \quad$ total active power generated at hour $h$

$P_{L}^{h} \quad$ total system active load at hour $h$

$P_{L_{i}}^{h} \quad$ active load from bus $i$ at hour $h$

$P_{\mathrm{PV}_{i}}^{h} \quad$ active power output of PV unit $i$ at hour $h$

$P_{S_{i}}^{h} \quad$ active power output of VSI unit $i$ at hour $h$

$Q \quad$ reactive power

$Q_{\text {step }} \quad$ reactive power step

$Q_{i}^{h} \quad$ reactive power output injected in bus $i$ at hour $h$

$Q_{L_{i}}^{h} \quad$ reactive load from bus $i$ at hour $h$

$Q_{\mathrm{MV}}^{h} \quad$ reactive power output from the medium voltage (MV) stage at hour $h$

$Q_{\text {out }} \quad$ reactive power output of the inverter

$Q_{\mathrm{PV}_{i}}^{h} \quad$ reactive power output of PV from bus $i$ at hour $h$

$Q_{S_{i}}^{h} \quad$ reactive power output by the storage unit (VSI) from bus $i$ at hour $h$

$\mathrm{SoC}_{i}^{h} \quad$ state of charge of VSI $i$ at hour $h$

$V \quad$ voltage at the inverter terminals

$V_{0} \quad$ voltage's idle value at no load conditions

$V_{\text {step }} \quad$ voltage step

$V_{i}^{h} \quad$ voltage magnitude in bus $i$ at hour $h$

\author{
$V_{i \mid Q=Q_{i}^{h}}^{h} \quad$ voltage magnitude in bus $i$ at hour $h$ for a reactive power \\ injected in bus $i$ at hour $h$ \\ $V_{\text {int }} \quad$ VSI internal bus voltage \\ $V_{\mathrm{MV}}^{h} \quad$ voltage magnitude from the MV stage at hour $h$ \\ $\alpha \quad$ cost of efficiency for (dis)charging storage units \\ $\beta \quad$ cost of power curtailment for PV units \\ $\eta_{C_{i}}, \eta_{D_{i}}$ charging/discharging efficiency of a storage device \\ associated with VSI unit $i$ \\ $\theta_{i}^{h} \quad$ voltage angles in bus $i$ at hour $h$ \\ $k_{P}, k_{Q} \quad$ active/reactive power droop slope \\ $\omega \quad$ angular frequency's at the inverter terminals \\ $\omega_{0} \quad$ angular frequency's idle value at no load conditions
}

\section{Introduction}

\subsection{Motivation and incitement}

Several factors have been driving large-scale integration of renewable energy sources into the electricity grid. This trend has led to innovative concepts, such as microgrids (MGs) and multiMGs (MMGs), which are seen as enabling solutions to the operational planning and control of future distribution systems with improved resilience [1-5].

MGs are typically regarded as low-voltage (LV) distribution systems aggregating loads, small-scale generation systems, which are generally designated as microsources (MS), and energy storage devices, being controlled and managed through a hierarchical arrangement supported by a communication infrastructure $[1,6]$. Following this view, MMG consists on a natural evolution of the MG concept: it aggregates MMG, distributed generation (DG) and other resources at the medium-voltage (MV) distribution level while exploiting also a centralised control and management system $[1,2]$. Considering the large-scale deployment of photovoltaic (PV) generation and energy storage devices in LV distribution grids, it is possible to assume a PV-battery MG and the clustering of those MGs at the MV level, thus forming a MG cluster (MGC), which can be regarded as a MMG. 
A distinctive characteristic of the MGC is related to its ability to operate autonomously in the face of an emergency or planned scenarios. However, system survival and its stability conditions largely rely on energy storage devices in order to provide proper frequency and voltage regulation $[1,6,7]$. Active power-frequency $(\mathrm{P}-\mathrm{f})$ and reactive power-voltage $(\mathrm{Q}-\mathrm{V})$ droop functions are fundamental real-time control features of power electronic interfacing energy storage devices to the grid. These control concepts are capable of providing a grid forming operation mode to these units thus enhancing its ability to act as a power buffer for continuous system balance during islanding operating conditions. While in autonomous operation mode, the system balance relies on the aforementioned droop controls acting as a primary control layer, which is responsible for primary frequency and voltage regulation. Nominal frequency restoration and bus voltage profiles maintenance are achieved through the dispatch of the existing resources by the secondary control layer $[8,9]$.

On top of these real-time control features, the islanded operation of a MGC must be planned beforehand to guarantee an adequate continuity of supply. Most of the DGs in MGs are variables in nature, which requires the definition of the optimal storage levels so that the excess generation in some periods can be used in others with generation deficit. This optimisation task must take into account the available resources in the MGC, which may involve energy exchange among MG in the cluster throughout the planning horizon.

The MGC operational planning for islanded operation is typically carried out by a centralised algorithm that requires the complete knowledge of the loads, the generation, and the storage devices. While resulting in the best operational plan, this type of algorithm poses several barriers to the complete deployment of the MGC framework, namely in terms of modularity, scalability, and interoperability. In fact, the centralised algorithm needs to be structurally updated each time a new MG is integrated into the MGC. In addition, data confidentially might not be completely assured since the location and characteristics of the electrical equipment in the MG must be disclosed to the centralised algorithm.

The work presented in this study tackles this problem by proposing a hierarchical optimisation strategy that enables an effective coordination of the resources available for MGC islanded operation in terms of active power exchanges and volt/var control while limiting the information exchanged between the MG and the central controller at the MMG level.

\subsection{Literature review}

A centralised approach based on the unit commitment and optimal power flow (OPF) formulation is proposed in [10] to solve the energy management problem of unbalanced MG. In [11], a twolevel optimisation process relying on multi-period energy management for clustered wind-PV-battery MG systems connected to the distribution network is presented. An approach for energy management based on multi-agent systems that follows a three-step hierarchical operation of a MMG system is suggested in [12]. In [13], the authors present an optimisation-based method for local power transactions and coordinated dispatch of storage devices aiming to reduce electricity cost in grid-connected MG. The proposed approach can be implemented over existing power networks (adding only a simple data communication infrastructure). A bi-level structure for integrating and managing various distributed resources within a MMG environment is proposed in [14]. This bi-level formulation is presented as an improved solution when compared to an alternative where a single entity controls the distributed resources. Amini et al. [15] employed a non-centralised strategy for optimal power routing based on cloud-computing in clustered direct current (DC) MG. Since each MG does not require information about the others, this concept can further incorporate additional MG, which makes it highly scalable. However, since the scope of this work only encompasses DC systems, it does not include the volt/var control problem. The other existing methods take into consideration a gridconnected operating system and/or adopt traditional approaches based on centralised control architectures, which do not take advantage of the hierarchical/distributed nature of the control architecture developed within the MMG framework.

\subsection{Contributions}

The main contributions of this study consist of the hierarchical optimisation strategy running at the MGC control infrastructure, which addresses the volt/var control problem and provides a multitemporal energy dispatch for an alternate current PV-battery MGC while operating islanded from the main grid. As previously mentioned, the strategy allows for a greater degree of flexibility and a more modular, interoperable and scalable characteristic, since it is based on a minimum flow of information between the MG and the MGC control infrastructure.

The effectiveness of the proposed strategy is demonstrated in a case study based on a MGC with two voltage levels $(15 \mathrm{kV}$ and $400 \mathrm{~V}$ ) and three interconnected LV MGs. The simulation results show that the proposed approach is capable of producing similar results to a centralised algorithm in terms of active power exchanges and voltage levels for a $24 \mathrm{~h}$ planning horizon. However, the approach leads to slightly higher active power losses (in this case $4.3 \mathrm{kWh}$ higher) when compared to a centralised algorithm due to its inability to optimise the active and reactive power simultaneously, which is the case of the fully centralised algorithm.

\subsection{Organisation of paper}

The remainder of the paper is organised as follows. Section 2 presents the MG and MMG concepts and framework as a baseline for the MGC environment. Section 3 demonstrates how the autonomous operation of a power system dominated by electrical converters is achieved. The conceptualisation and design of the proposed approach are explained in Section 4. Section 5 describes the case study and Section 6 discusses the obtained simulation results and the achievements of the proposed strategy. The main ideas of the paper are summarised and the conclusions are presented in Section 7.

\section{MG and MMG}

\subsection{MG concept, control architecture and operation modes}

The CIGRE (International Council on Large Electric Systems) working group WG C6.22 provided a clear definition of MG, stating that 'microgrids are electricity distribution systems containing loads and distributed energy resources, (such as distributed generators, storage devices, or controllable loads) that can be operated in a controlled, coordinated way either while connected to the main power network or while islanded' [16].

The extended degree of flexibility envisioned for MG requires proper management and control architectures supporting key functionalities in both normal interconnected and islanded operation modes, being supported by specific communication infrastructures. The MG central controller (MGCC) leads the upper level of management and control in a MG. This controller, usually housed in the LV side of the MV/LV transformed, coordinates the MS and storage controllers [microsource controller (MC)] as well as the load controllers (LCs) (Fig. 1). MCs are embedded in the power converters associated with MS and storage devices. LCs regulate a group or individually controllable loads. According to a pre-determined method, the MC and LC are fed operation setpoints by the MGCC $[1,6,17]$.

\subsection{MMG concept, control architecture and operation modes}

The high penetration of DG in MV distribution grids and the development of the MG concept lead to the need for extending it to a broader context. In this sense, the concept of a MMG can be regarded as a structure of interconnection between the MV network to LV MG and DG, which is also actively controlled and managed by a hierarchical system $[2,17]$. 


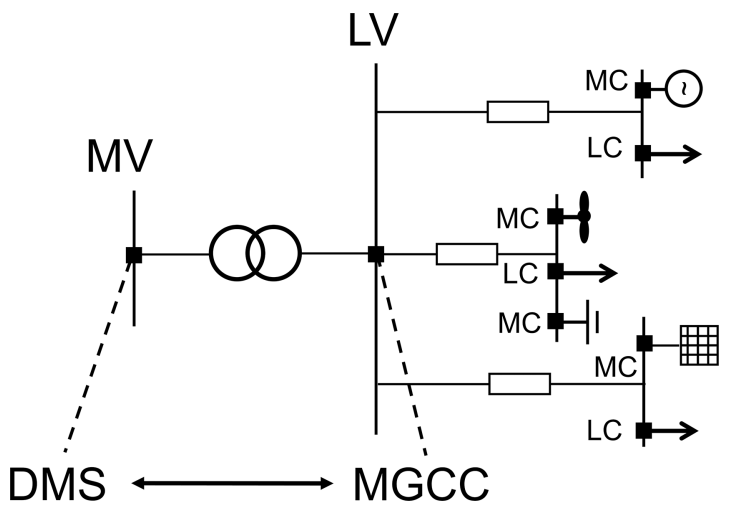

Fig. $1 M G$ architecture [1]

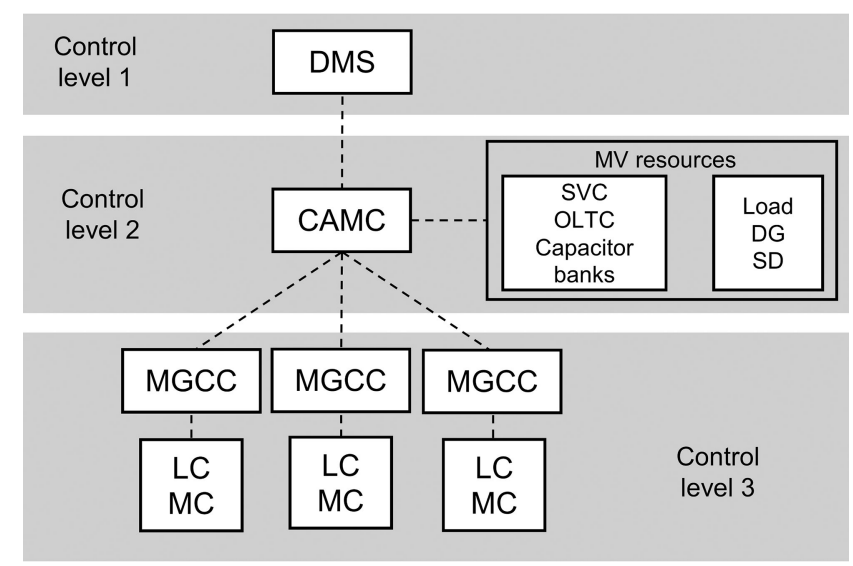

Fig. $2 M M G$ control scheme [17]
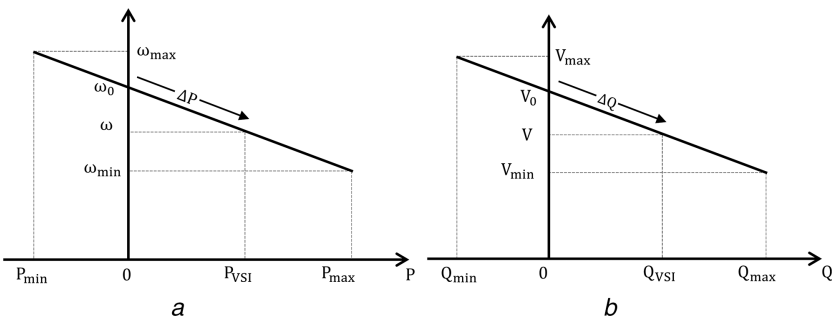

Fig. 3 VSI droop characteristics

(a) P-f droop, (b) Q-V droop [18, 19]

The concept of MMG is related to a higher-level structure than a MG and includes an additional management agent, the central autonomous management controller (CAMC), as depicted in Fig. 2. This controller is housed in the high-voltage to MV distribution substation and is responsible to collect data from lower rank controllers (such as MGCC and MV level controllers for DG units and loads connected directly to the MV grid) and establish rules for its operation [1,17]. This control and management system can be regarded as a three-level hierarchical structure as is presented in Fig. 2. In level 1, the distribution management system (DMS) is in charge of the management of the whole distribution grid. The CAMC represents the second level of autonomy in the distribution grid chain and is responsible for managing MV level operation within the MMG concept by interacting with MV-connected resources such as the MGCC (that aggregates downstream other elements, as previously described), as well as MV-connected resources such as loads and DG units. At level 3, the LV network is managed and controlled by a MGCC that coordinates and manages the operation of several devices such as MC and LC in close coordination with the CAMC [17]. Therefore, the MMG concept and control architecture intrinsically constitutes the seminal reference for the operation of a MGC.

Similarly to MG, MMGs are able to operate in normal interconnected or in autonomous/emergency mode. In the first mode, MMG operates connected to the main distribution grid. The
CAMC establishes the operation set-points of MG (through the associated MGCC) and other MV-connected resources in close coordination with the DMS [9]. In this operation mode, the CAMC takes the responsibility of globally optimising the MMG with respect to certain objectives: minimising power losses, coordinating power exchanges between $\mathrm{MG}$ and improving voltage profiles [17].

Secure and successful islanding of a MMG, subsequently to a disturbance in the upstream grid, requires the proper coordination of frequency control mechanisms by the CAMC. In this primary type of control, the CAMC is responsible for management and regulation of all MGCC, controllable loads and DG directly coupled to the MV network. The secondary control layer for autonomous operation acts identically to the classical automatic generation control. For a pre-defined period, the steady state frequency error (or deviation from the nominal value) is used to compute and send new operation set-points by the CAMC to the controllable resources under its control [9].

\section{Enabling autonomous operation}

The autonomous operation of $\mathrm{MG}$ or MGC requires the implementation of suitable control features for both frequency and voltage. These functionalities can be categorised as primary and secondary layers of control [18].

Since most of the MS connected to LV grids and DG units connected to the MV grid, as well as energy storage devices, have power electronics interfaces and also considering a converter dominated grid without any synchronous generator connected, the primary control level relies on the two essential control methods for those converters: grid-tied (or PQ) and grid-forming [or voltage source inverter (VSI)].

The first type of control (PQ or grid-tied inverter control) allows for power converters to synchronise with an energised grid and establishes operational set-points in terms of active and reactive power. The PQ inverter acts as a current-controlled voltage source that maintains synchronism with the grid [19]. Given this feature, this type of inverters' control is typically employed in the converter interfaces available in renewable-based sources such as solar or wind power [6].

The VSI or grid-forming inverter control mode is usually implemented for the converters associated with energy storage units since they are capable of rapidly reacting to sudden load changes, thus performing as voltage sources. In principle, an autonomous system requires at least one unit of this type, which intrinsically has the ability to form the grid voltage such that autonomous operation is possible. The voltage and frequency control feature present in this type of inverter is achieved through the implementation of the subsequent droop functions $[6,19]$

$$
\begin{aligned}
& \omega=\omega_{0}-k_{P} \times P, \\
& V=V_{0}-k_{Q} \times Q .
\end{aligned}
$$

According to the P-f droop function in (1) and Fig. $3 a$, the frequency deviation from the nominal value is proportional to the load increase. Similarly, with the $Q-V$ droop function in (2) and Fig. $3 b$ as the reactive power loading increases the voltage at VSI terminals decreases and vice-versa. This control principle allows VSI to respond automatically based only on the information available at its terminals $[1,20]$. Also, it allows for active power sharing among a set of VSI according to the P-f droop since grid frequency is a global variable. Nevertheless, due to the voltage drops along the lines, reactive power sharing according to the Q-V droop is not possible to be achieved without a supplementary control level.

The continuous islanded operation of MG and MGC requires implementation of secondary-type control functionalities in order to guarantee the dispatch of the existing resources [18] while assuring frequency and voltage recovery to the defined settings. There were identified several types of secondary control strategies which could be divided into two groups: a local control implemented in every $\mathrm{MC}$ and a centralised type of control 


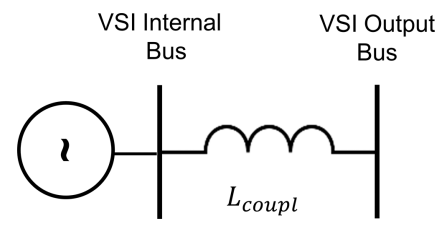

Fig. 4 VSI model for power flow studies [19]

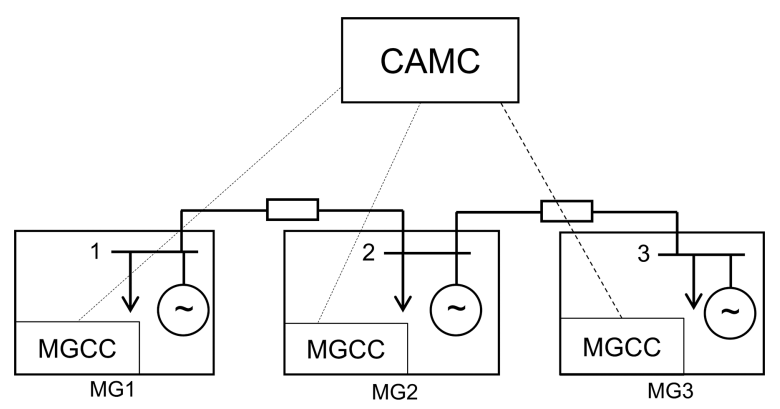

Fig. 5 MGC diagram

coordinated by the CAMC and the MGCC $[4,18]$. As previously discussed, the secondary control dispatches DG and MG-connected resources sending active power set-points with the objective of recovering nominal system frequency $(50 \mathrm{~Hz})$. Assuming the effectiveness of the secondary frequency control, it can be considered that the frequency remains at its nominal value and steady state conditions are achieved. Under these circumstances, solving beforehand the load flow problem in the autonomous system is crucial to the development of any management strategy.

\subsection{Power flow during autonomous operation}

The use of a droop-based control approach as an enabling mechanism for autonomous operation of the system precludes the proper sharing of reactive power among existing VSIs as a result of the line voltage drops. It is also necessary to consider that P-f (1) and $\mathrm{Q}-\mathrm{V}$ (2) droop functions were designed for a high inductive coupling to the grid, allowing for the decoupling between $\mathrm{P}$ and $\mathrm{Q}$. However, the feeders in LV systems (such as MG) have a predominant resistive component (high $R / X$ ratio), which makes the coupling of droop-based inverters highly resistive. As a result, active power influences the voltage magnitude value which contradicts the classical approach of reactive power control over voltage and $\mathrm{P}-\mathrm{Q}$ decoupling [19]. To overcome this issue, there are many techniques and methods present in the literature, such as the ones presented in $[7-9,21]$, in order to assure the stable operation of droop-controlled inverters in autonomous systems. Several of these references exploit the concept of virtual impedance to be implemented in electronic power converters. The application of this concept on grid-forming inverters (or VSI) droop control can artificially improve $\mathrm{P}-\mathrm{Q}$ decoupling by virtually changing these inverters output impedance in order to become more inductive [8, 9,21].

A droop-controlled VSI can be viewed as a voltage source connected to the grid by a coupling inductance that corresponds not only to the physical reactance but also to the virtual impedance value added by the control system. Following this, in power flow studies, the VSI is represented by two buses (Fig. 4): an internal bus between the voltage source and the coupling inductance and another bus that acts as the interface with the grid (output bus) [19].

In agreement with the Q-V droop function (2), controlling voltage in the VSI output bus depends on the reactive power loading at its terminals. Since $k_{Q}$ is a fixed characteristic of each VSI, the appropriate setting of the idle voltage $V_{0}$ allows for reactive power and voltage control [19]. From the power flow studies model, the idle voltage $V_{0}$ can be computed as

$$
V_{0}=V_{\text {int }}+k_{Q} \cdot Q_{\text {out }} .
$$

\section{Problem formulation}

The MGC follows the MMG concept: it is formed within a MV distribution grid that interconnects several LV MGs as depicted in Fig. 5. Each MG comprises a LV distribution grid, loads, PV generation and energy storage devices coupled to the grid through a VSI.

In the event of sudden islanding of a cluster of MG (islanding taking place at the MV grid level), it is necessary to introduce some management capability of the available resources (in this case, energy storage systems and PV) such that proper autonomous system operation can be achieved, as described in Section 2 .

Following the MMG concept, the MGC is centrally managed by a CAMC responsible for the technical management of the MV grid level. This upstream controller interacts with downstream controllers associated with each MG (the MGCC). Within each MG, controllers associated with PV systems and energy storage devices, as well as with loads, interact with the MGCC. Such an operation framework requires two main sources of information, namely load and PV power generation forecasts for the upcoming time steps (depending on the horizon it is expected the MGC to operate autonomously). This information is provided at the MGCC level, is then communicated to the CAMC responsible for managing the MGC.

The energy scheduling and volt/var control in islanded systems is a key functionality in the islanded mode of operation for addressing power scheduling while maintaining voltage profiles within an acceptable range following the reaction of the primary control mechanisms [18]. Since energy storage devices have inherent capacity limits, the underlying management problem has a multi-temporal nature, being necessary to take into account the state of charge of storage devices derived from previous periods of operation, and the grid's present and future demand as well as the present and future availability power production (from DG).

\subsection{Conceptual approach}

The proposed approach is intended to ensure the proper scheduling of active power and volt/var control within a MGC operating autonomously such that secure operation is achieved. It is responsible for determining, a priori, an operation strategy for the next few hours (e.g. $24 \mathrm{~h}$ ), based on load and microgeneration forecasting (input information that must be made available by other tools that are out of the scope of this work) and considering the energy capacity range of distributed energy storage units available in the different MGs, as well as the possibility of allowing power exchanges in order to enable mutual support among the MG interconnected within the cluster.

The proposed strategy encompasses two main stages, as depicted in the flowchart from Fig. 6. In the initial stage, while considering the forecasted generation and consumption for a given period of time (e. g. the expected duration of the islanded operation), an initial optimal active power dispatch is solved for the overall MGC without considering the grid constraints. This initial problem includes the multi-temporal constraints as a result of the charging state of the energy storage devices. In the second stage, the reactive power scheduling and volt/var control problem in the MGC are addressed and solved in a coordinated way between the CAMC and the MGCC.

\subsection{Sequence of events and communication flow}

The proposed algorithm is intended to run within the MGC architecture, thus enabling a hierarchical/distributed, modular and scalable approach to the problem while complying with the desired plug-and-play characteristic that is intended for this type of system [22]. Under the proposed approach for the scheduling problem, the CAMC does not need to previously hold all the detailed information of the complete MGC network. Each individual MGCC provides the necessary data to the CAMC to start and run the proposed algorithm. As shown in Fig. 7, the MGCC computes the load and PV generation forecasts for the envisioned horizon of autonomous operation which are then communicated to the CAMC. After receiving all relevant data, the CAMC performs a 


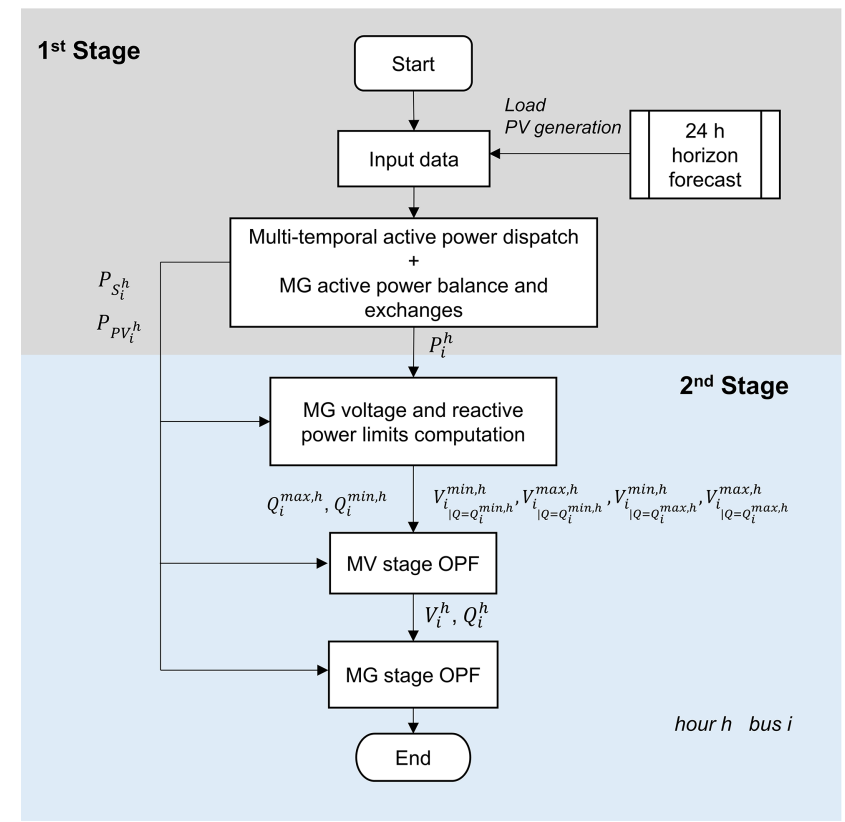

Fig. 6 Proposed algorithm flowchart

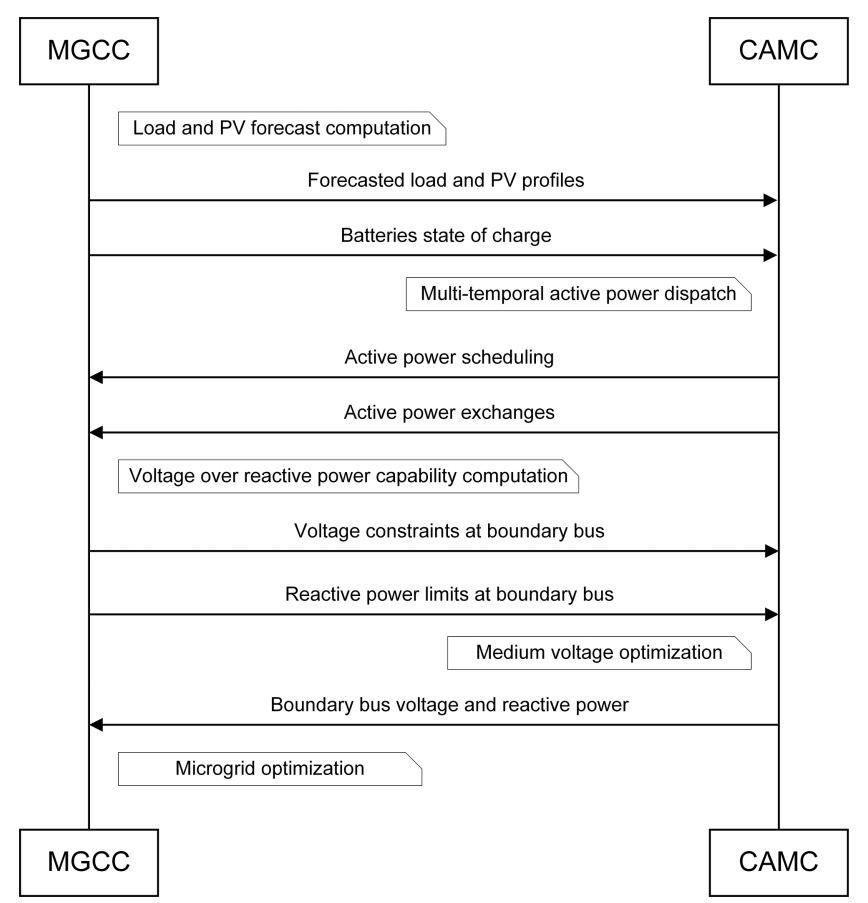

Fig. 7 Sequence of events and information flow

multi-temporal active power dispatch for the cluster. The active power set-points and the exchange of active power expected to occur are communicated to the respective MGCC. With this information, each MGCC computes the voltage and reactive power capability constraints (i.e. $V(Q)$ capability area) for the boundary bus. Note that this task can run in parallel at each MGCC, mirroring the distributed natured of the physical MGC control system. The boundary bus of each MG is considered to be its MV connection point with respect to the cluster. When this procedure is concluded for every time step and MG, the CAMC is informed by the MGCC about the reactive power capability at each boundary bus and performs an optimisation algorithm that adjusts the reactive power in the MV side of the network. The resulting boundary bus voltage and reactive power flows are then transmitted to each MGCC. Finally, taking into account these volt/var operation points for the boundary buses, each MGCC performs an internal optimisation (for every time step) in order to schedule their own operation as a function of the available resources per time step.

\subsection{Multi-temporal active power dispatch}

In this multi-temporal optimisation problem, the objective function $(\mathrm{OF})(4)$ is defined as the minimisation of charging and discharging energy associated with the storage devices. This OF is subjected to a set of technical constraints, namely: system active power balance constraint for each time step (5), energy storage devices' state of charge constraint (6), which introduces a multi-temporal characteristic to the problem, PV power curtailment constraint (7) and technical limit constraints for power and energy associated with each energy storage device (8)-(10)

$$
\min \sum_{h=1}^{H} \sum_{i=1}^{N_{S}}\left(\alpha \cdot\left(P_{D_{i}}^{h}+P_{C_{i}}^{h}\right)+\beta \cdot P_{\mathrm{Cur}_{i}}^{h}\right) .
$$

Subject to

$$
\begin{gathered}
\sum_{i=1}^{K} P_{\mathrm{PV}_{i}}^{h}+\sum_{i=1}^{N_{S}} P_{D_{i}}^{h}-\sum_{i=1}^{N_{S}} P_{C_{i}}^{h}=P_{L}^{h}, \\
\mathrm{SoC}_{i}^{h}=\mathrm{SoC}_{i}^{h-1}+\left(\eta_{C_{i}} \cdot P_{C_{i}}^{h}-\frac{P_{D_{i}}^{h}}{\eta_{D_{i}}}\right), \\
0 \leq P_{\mathrm{Cur}_{i}}^{h}<P_{\mathrm{PV}_{i}}^{\max }, \\
P_{D_{i}}^{\min } \leq P_{D_{i}}^{h} \leq P_{D_{i}}^{\max }, \\
P_{C_{i}}^{\min } \leq P_{C_{i}}^{h} \leq P_{C_{i}}^{\max }, \\
\mathrm{SoC}_{i}^{\min } \leq \mathrm{SoC}_{i}^{h} \leq \mathrm{SoC}_{i}^{\max } .
\end{gathered}
$$

After solving this problem, it is possible to obtain scheduling for the active power use from energy storage devices and potential active power curtailment in PV systems in the case of overproduction. This initial solution provides also information about the active power operation regime of each $\mathrm{MG}$ within the cluster, as well as the power imports/exports among the existing MG. The next step is related to the voltage and reactive power management of the MGC, taking into account the active power scheduling for each time step.

\subsection{Voltage and reactive power control}

As it was previously stated, VSI units connected to the energy storage devices and operated in the voltage-reactive power droop mode are responsible for the real-time volt/var control problem in the MGC in a distributed manner, which can be complemented with the reactive power support capability from PV sources. Given the coupling among the MG within the cluster, it is first necessary to properly schedule the reactive power transfers among them from the MV level.

This problem can be managed by the CAMC exploiting a proper approach (MV optimisation stage), which needs to be fed from the MG level (through the MGCC) with proper information: the voltage and reactive power capability limits for each time step (it is assumed this information is provided at the boundary bus of each MG, which is defined as the MV connection point of each MG to the cluster). Hence, a LV optimisation procedure running at the MGCC level can be performed in order to define a feasible volt/var area of operation within the MG, taking into account the active power constraints defined at the first optimisation stage.

4.4.1 Voltage and reactive power capability limits for the boundary bus of each MG: The identification of the voltage and reactive power capability of each $\mathrm{MG}$ is to be performed at the MGCC level of each MG. For every time step and MG, there is a maximum and minimum limit to the reactive power output for each voltage level that is possible to be achieved at the boundary bus. To determine those constraints, the algorithm presented in Fig. 8 is used for each time step. The procedure consists of fixing the active 


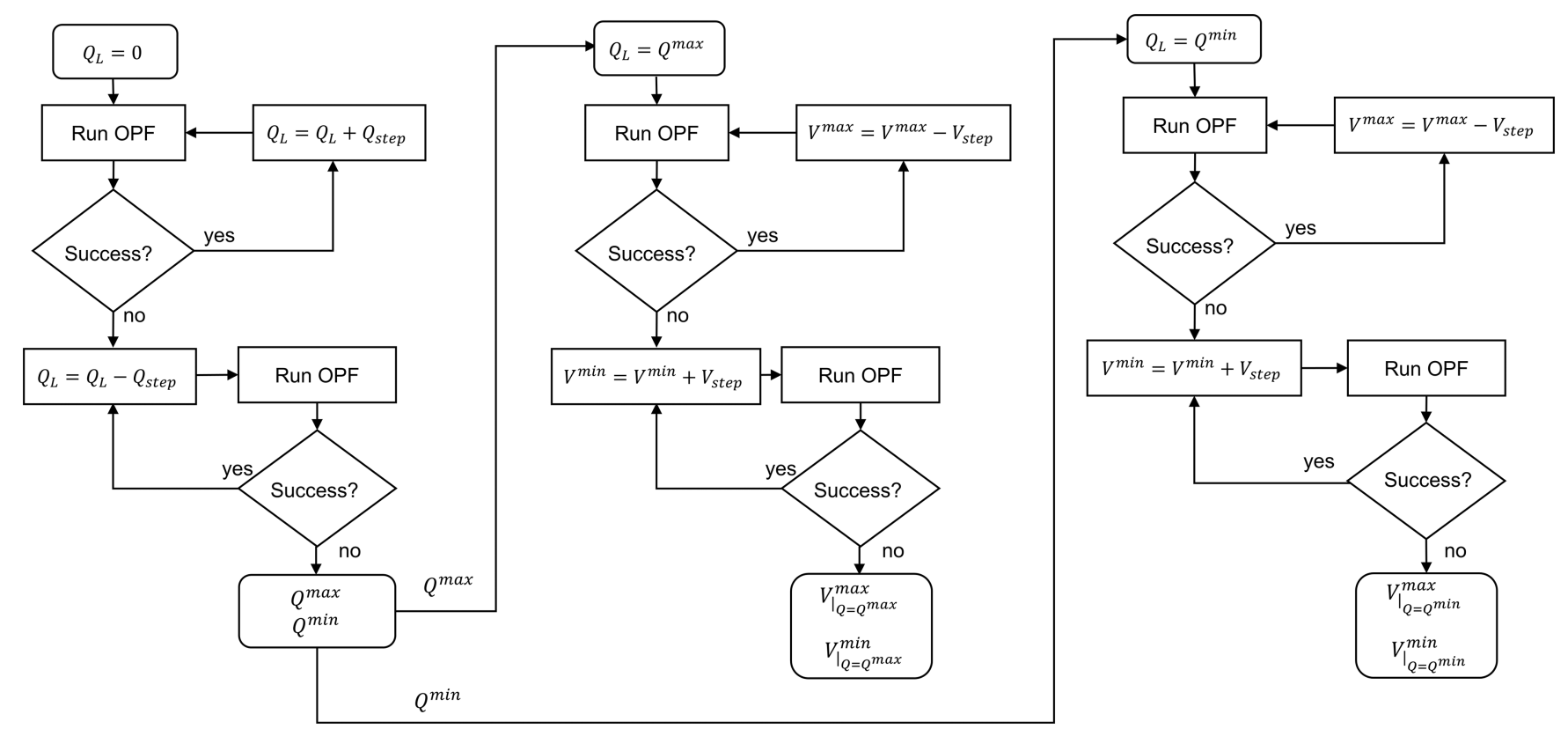

Fig. 8 V/Q limits computation for a specific $M G$ and hour

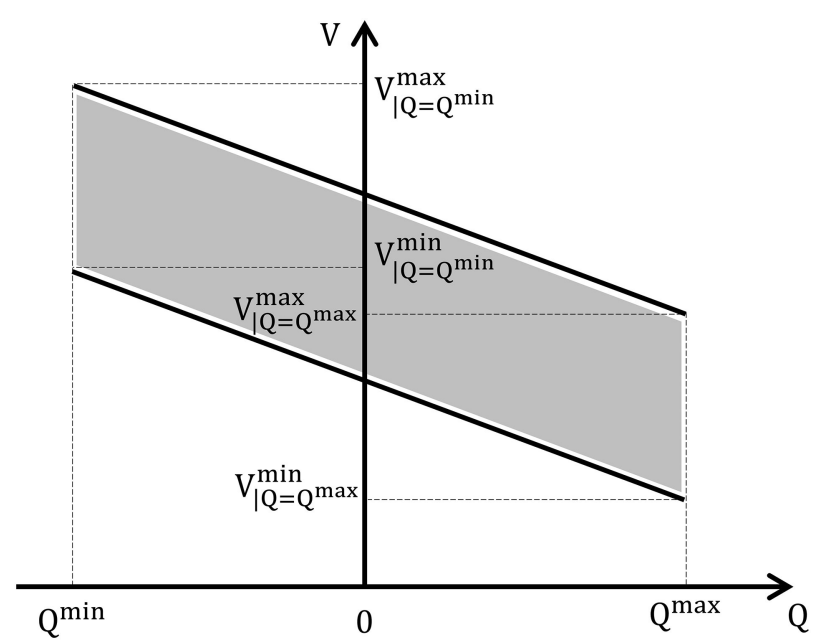

Fig. $9 V(Q)$ capability area shaded in grey for a specific $M G$ and time step

power at the MG boundary bus for each time step and change (in pre-defined step magnitudes) the reactive power (injected/ consumed) at that bus up to a feasible limit. The reactive power limits are defined by the successful run of a MG-level OPF that is responsible to find out a feasible solution for reactive power dispatch within the MG. After finding these reactive power limits, this simple but effective algorithm computes the voltage restrictions by considering the reactive power fixed at the boundary bus. Note that this algorithm can be based on balanced or unbalanced models regarding the operating conditions of each MG. In this work, and without compromising the main contribution presented, balanced operation of the MG is assumed. As depicted in Fig. 8, the reactive power at the MG boundary bus starts from zero and is progressively increased up to a feasible limit (defines the maximum injection of reactive power from the MG to the cluster and the corresponding voltage at the boundary bus). Then, the reactive power at the boundary bus is decremented progressively from zero in order to identify the minimum injection of reactive power from the cluster to the MG. With the reactive power maximum and minimum defined, two similar procedures are then run in parallel: one to find the voltage range at the boundary while the reactive power injected in it is fixed at its maximum and another one to find the voltage at the boundary while the reactive power injected in it is fixed at its minimum. This procedure results in four points of operation that correspond to the corners of a volt/var $(V(Q))$ convex capability area of operation that is generically presented in Fig. 9. Note that there are other possible optimisation methods to compute the $V(Q)$ capability area. In addition, the true $V(Q)$ capability might take other forms, depending on the MG. In this method, and for the sake of the optimisation process run at the CAMC level, we only require the area to take a convex shape. This information is then used at the MV stage optimisation in order to define the constraints of the optimisation problem for reactive power coordination among the MG.

4.4.2 MV reactive power adjustment: At this stage, the CAMC can be informed from the MGCC about active power flows to be established among MG within the cluster, as well as the reactive power and voltage constraints at each MG boundary bus. As a result, each boundary bus is assigned with an active power value for each time step, reactive power, and voltage restrictions. Therefore, the key issue is then to define the reactive power at the boundary bus that is to be supplied by each MG. In this case, for a particular time step, an OPF-like approach is performed with the objective of minimising active power losses while constrained by the voltage and reactive power constraints as defined by (12)-(14).

$$
\min P_{\text {Losses }}^{h} \text {. }
$$

Subject to

$$
\begin{aligned}
& Q_{i}^{\min , h} \leq Q_{i}^{h} \leq Q_{i}^{\max , h},
\end{aligned}
$$

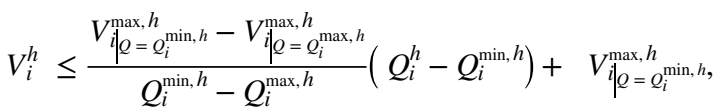

$$
\begin{aligned}
& V_{i}^{h} \geq \frac{V_{i \mid Q=Q_{i}^{\min , h}-V_{i}^{\min , h}, h=Q_{i}^{\max , h}}}{Q_{i}^{\min , h}-Q_{i}^{\max , h}}\left(Q_{i}^{h}-Q_{i}^{\min , h}\right)+V_{i \mid Q=Q_{i}^{\min , h} .}^{\min ,}
\end{aligned}
$$

4.4.3 MG stage: After performing the MV grid optimisation at the CAMC level, the final step is developed at the MG level. This stage is intended to compute a feasible solution at the MG level through an OPF-like approach based on Meirinhos et al. [23] and similar to those of Sun and Zhang [24], taking into account its internal capabilities for voltage and reactive power support (PV reactive power generation and idle voltage definition at each VSI while also complying with the boundary bus operation point defined at the MV stage. Note that (15) corresponds to the minimisation of active power losses in the MG and it is subjected to the power balance constraints for each node as in (16) and (17), 


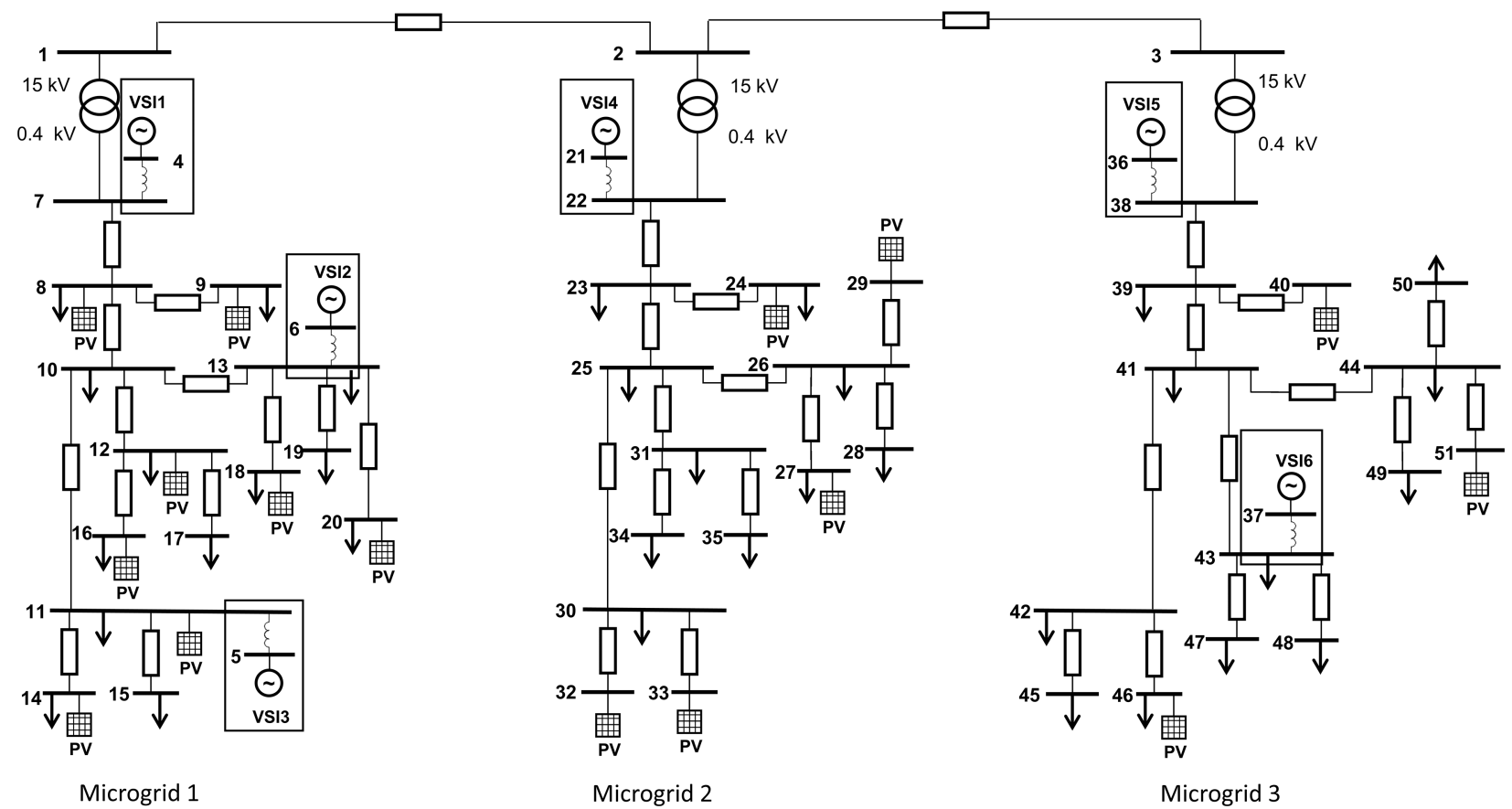

Fig. $10 M G C$ single line diagram

Table 1 Test system composition

\begin{tabular}{lccccc}
\hline MG & Buses & Branches & VSI & PV & Loads \\
\hline 1 & 18 & 16 & 3 & 8 & 13 \\
2 & 16 & 14 & 1 & 5 & 10 \\
3 & 17 & 15 & 2 & 3 & 11 \\
\hline
\end{tabular}

bus voltage constraints in (18) and (19), reactive power constraint in the swing bus (18) and devices technical constraints in (20)(22). The MG operation state that results from this stage corresponds to the final solution for reactive power and voltage control for the MGC. Since the initial active power dispatch neglected network constraints, some active power adjustments are due to the VSI acting as the swing bus at each MG in this stage in order to compensate for the active power losses

$$
\min P_{\text {Losses }}^{h} \text {. }
$$

Subject to

$$
\begin{gathered}
P_{\mathrm{PV}_{i}}^{h}+P_{D_{i}}^{h}-P_{C_{i}}^{h}-P_{L_{i}}^{h} \\
=V_{i}^{h} \cdot \sum_{k=1}^{N_{B}} V_{k}^{h} \cdot\left(\begin{array}{l}
G_{i k} \cdot \cos \left(\theta_{i}^{h}-\theta_{k}^{h}\right) \\
+B_{i k} \cdot \sin \left(\theta_{i}^{h}-\theta_{k}^{h}\right)
\end{array}\right), \\
Q_{\mathrm{PV}_{i}}^{h}+Q_{D_{i}}^{h}-Q_{C_{i}}^{h}-Q_{L_{i}}^{h} \\
=V_{i}^{h} \cdot \sum_{k=1}^{N_{B}} V_{k}^{h} \cdot\left(\begin{array}{l}
G_{i k} \cdot \sin \left(\theta_{i}^{h}-\theta_{k}^{h}\right) \\
-B_{i k} \cdot \cos \left(\theta_{i}^{h}-\theta_{k}^{h}\right)
\end{array}\right), \\
V_{i}^{\min , h} \leq V_{i}^{h} \leq V_{i}^{\max , h}, \\
V_{1}^{\min , h}=V_{\mathrm{MV}^{h}}^{h}=V_{1}^{\max , h}, \\
Q_{L_{1}}^{h}=Q_{\mathrm{MV}}^{h}, \\
Q_{S_{i}}^{\min } \leq Q_{C_{i}}^{h} \leq Q_{S_{i}}^{\max }, \\
-0.2 \cdot P_{\mathrm{PV}_{i}}^{h} \leq Q_{\mathrm{PV}_{i}}^{h} \leq 0.2 \cdot P_{\mathrm{PV}_{i}}^{h} .
\end{gathered}
$$

\section{Case study}

The case study considered to demonstrate the effectiveness of the proposed approach is based on a three-phase balanced operation of the test system from Fig. 10 in the Appendix. It consists of a MGC with two voltage levels (MV level at $15 \mathrm{kV}$ and LV level at $400 \mathrm{~V}$ nominal voltages). The test system comprises three LV MGs interconnected through $2 \mathrm{MV}$ lines. Each MG has a 15/0.4 kV transformer connecting the two voltage levels. The elements connected to each MG are summarised in Table 1. More details of the MGC studied are presented in the Appendix.

The MGC considered in this work is assumed to suddenly disconnect from the upstream distribution network at a certain moment and is expected to operate as such for the next $24 \mathrm{~h}$. For the autonomous operation period of the MGC system, it is required to determine the active power dispatch and to address the volt/var control problem. Thus, in Figs. 11 and 12, the forecasted profiles of PV generation and power consumption that were considered for each MG in the cluster are displayed. The PV production profiles were derived from records of three different locations of residential level solar installations in Portugal. The load profiles correspond to typical working days of Portuguese domestic consumers. These profiles allow for the testing of different operational scenarios, such as peak, valley and noon scenarios.

Within this MGC, it is also assumed the presence of six energy storage devices that will be referred as VSI and numbered sequentially (VSI1, 2 and 3 belong to MG1, VSI4 to MG2 and VSI5 and 6 to MG3). It was considered the same power capacity for all the power converters $\left(P_{\max }=50.0 \mathrm{~kW}, P_{\min }=-50.0 \mathrm{~kW}\right.$, $Q_{\max }=32.5 \mathrm{kVar}$ and $\left.Q_{\min }=-32.5 \mathrm{kVar}\right)$ and a typical (dis)charging efficiency of $80 \%$ [25]. Table 2 exhibits the energy capacity (upper and lower limits for each energy storage unit connected to a VSI), as well as the considered state of charge prior to islanding. 


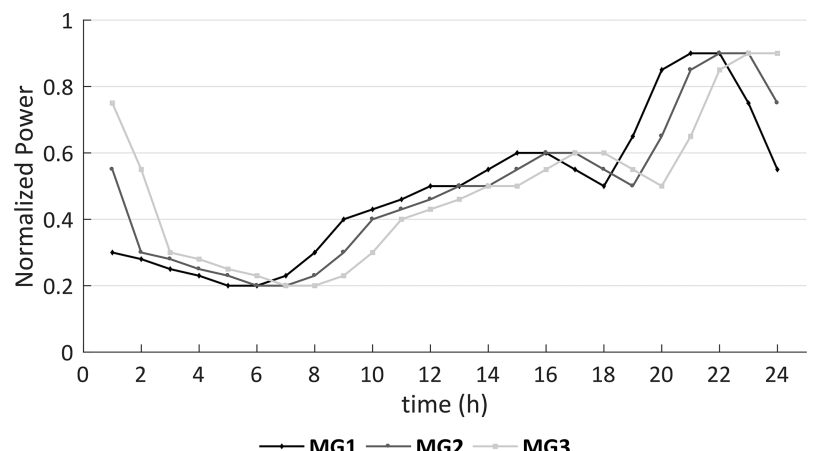

Fig. 11 Load profile forecast for each $M G$

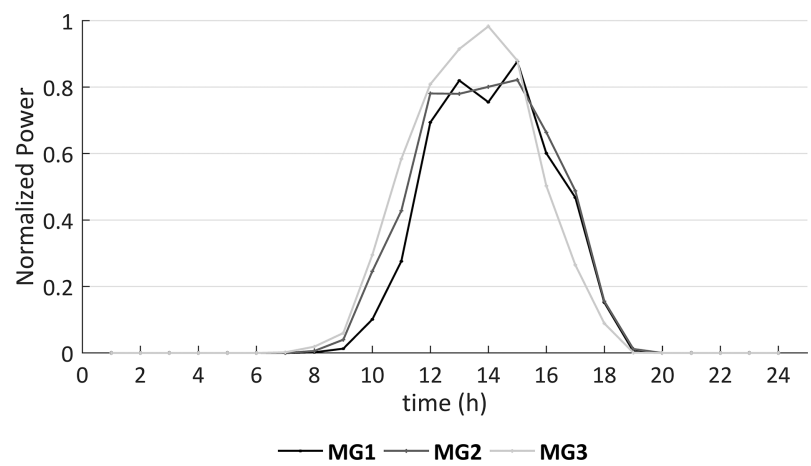

Fig. $12 P V$ generation profile forecast for each $M G$

Table 2 Energy storage devices capacity

\begin{tabular}{lccc}
\hline VSI & Max, kWh & Min, kWh & Initial, kWh \\
\hline 1 & 750.0 & 150.0 & 567.0 \\
2 & 625.0 & 125.0 & 472.5 \\
3 & 375.0 & 75.0 & 283.5 \\
4 & 750.0 & 150.0 & 567.0 \\
5 & 875.0 & 175.0 & 661.5 \\
6 & 1000.0 & 200.0 & 765.0 \\
\hline
\end{tabular}

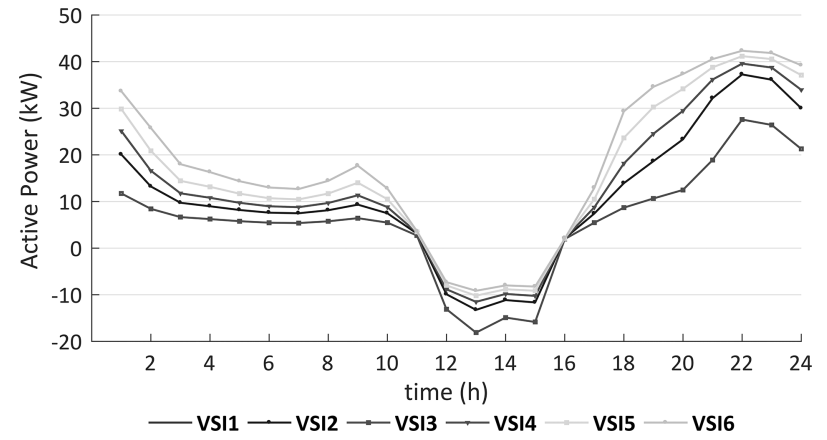

Fig. 13 VSI active power output schedule $(\mathrm{kW})$

The development of the suggested optimisation strategy was accomplished by applying MATPOWER's library [26], using the MATPOWER Interior Point Solver (MIPS) algorithm to solve the optimisation problems. The simulations were carried out on an Intel Core i7-2600 CPU @ 3.4 GHz with 8.0 GB of RAM PC in a MATLAB ${ }^{\circledR}(\mathrm{R} 2016 \mathrm{~b})$ environment.

\section{Obtained results and main achievements}

\subsection{Active power dispatch results}

6.1.1 VSI active power dispatch and state of charge in the energy storage devices: Figs. 13 and 14 depict, respectively, the active power dispatch for each VSI and state of charge of the corresponding energy storage device, during the autonomous operation period. It can be observed from Figs. 13 and 14 that all

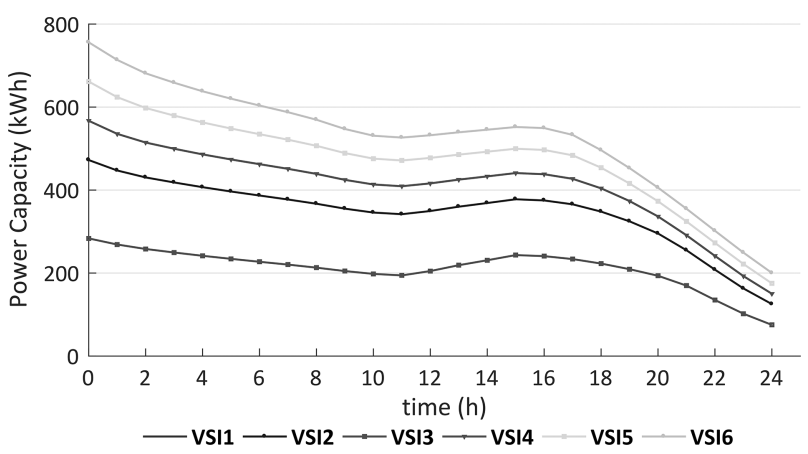

Fig. 14 Energy storage devices' state of charge ( $k W h$ )

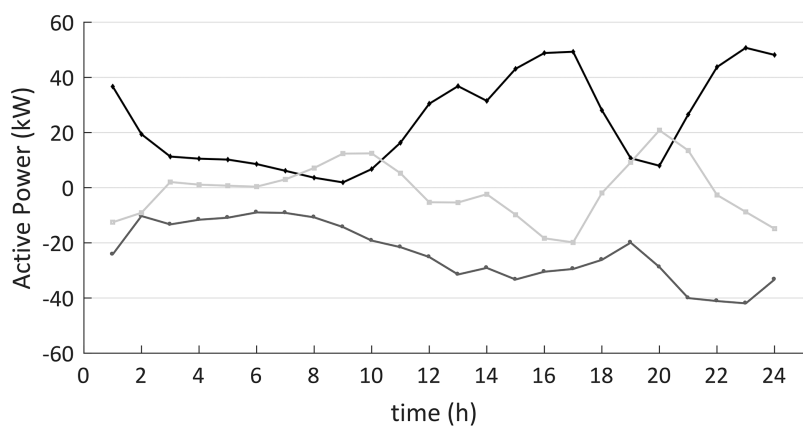

$\longrightarrow \mathrm{MG1}-\mathrm{MG2} \longrightarrow \mathrm{MG3}$

Fig. 15 Active power exchanges in the $M V$ grid

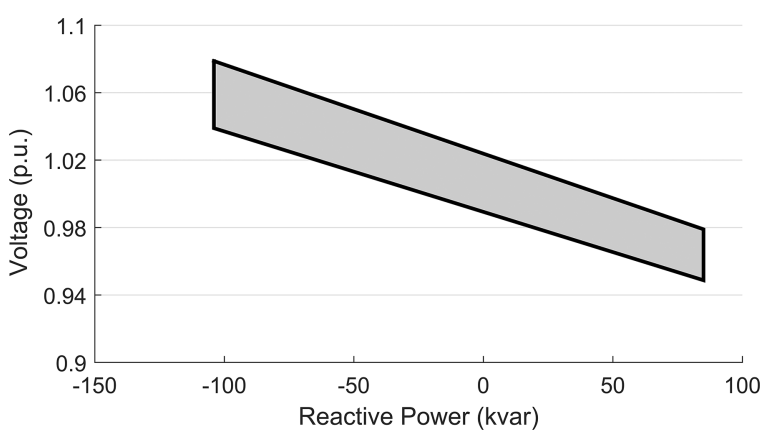

Fig. $16 V(Q)$ area for $M G 1$ and hour 6

VSIs are discharging (or outputting positive power) from hour 1 to 11 which means that there is no sufficient PV generation in the MGC to supply all loads. From hour 12 to 15 , the solar production rises to a point which exceeds the total load and so the storage devices charge. As the load increases (around hour 17), the VSI needs to discharge to support the system consumption needs. In addition, there was no need for PV power curtailment in the full $24 \mathrm{~h}$ horizon.

6.1.2 MG active power exchanges: After performing the active power dispatch, it is possible to estimate the active power transfers expected to occur among MG within the cluster. Fig. 15 shows that most of the time MG1 is capable of exporting energy to the others. In contrast, MG2 needs to import power during the full horizon of $24 \mathrm{~h}$ (a positive value means power export from a MG).

\subsection{MV stage results}

6.2.1 Reactive power exchanges and boundary bus voltage results: Figs. 16-18 present the obtained $V(Q)$ capability area of operation for MG1 for hours 6,13 and 18, respectively.

The area from Fig. 17 is noticeably larger than the ones from Figs. 16 and 18. This is a result of the higher reactive power availability $([-131.0,96.5] \mathrm{kVar})$ at this solar peak hour. This wider reactive power range reflects on an also larger range of permissible voltage magnitude values at MG1 boundary bus ([0.9006, 1.0780] p.u.). 


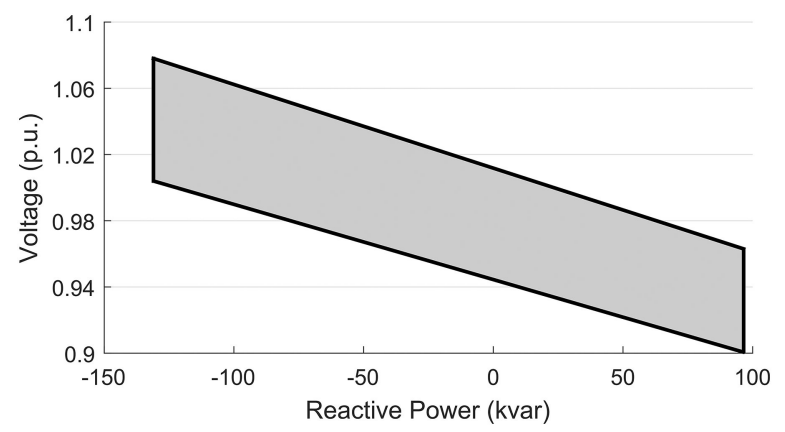

Fig. $17 V(Q)$ area for $M G 1$ and hour 13

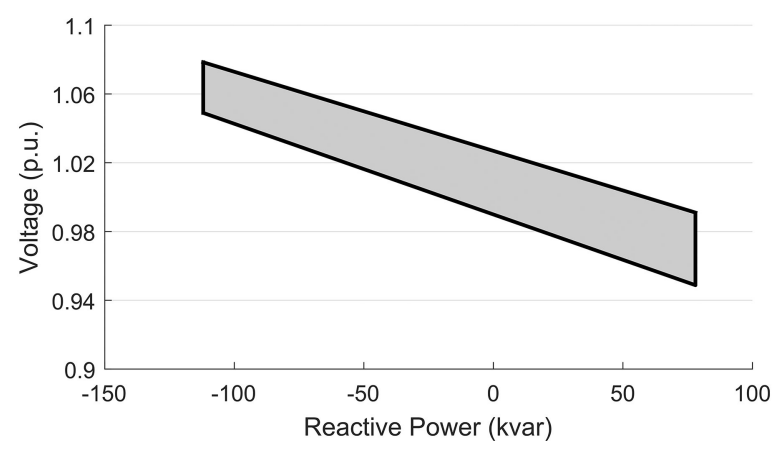

Fig. $18 V(Q)$ area for $M G 1$ and hour 19

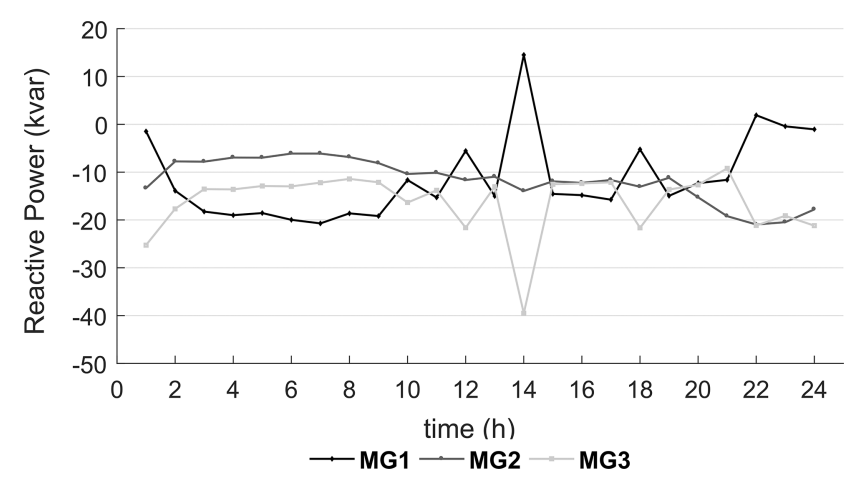

Fig. 19 Reactive power exchanges in the $M V$ grid

After computing the information about $V(Q)$ capability area, the algorithm performs the MV stage's OPF that result in the reactive power exchanges shown in Fig. 19. For most of the $24 \mathrm{~h}$ horizon, all MGs are importing reactive energy. This is because of the high susceptance $(B)$ of MV cables used as interconnectors among the MGs within the cluster. As for the boundary bus voltages, it seems that all of the three buses follow a similar magnitude value, which comes from the low resistive $(R)$ nature of MV feeders as well as from the reduced active power exchanges among the MG.

\subsection{Final results}

6.3.1 MG stage power dispatch: The final step of the proposed approach consists of defining a feasible solution at the MG level while considering the results from the MV stage for the boundary bus of each MG. At the same time, individual MG losses can also be evaluated since they were not considered in the initial stage of the approach. As shown in Figs. 20-22, the final active power scheduling is very close to the one initially defined. The difference between the initial and final curves corresponds to the active power losses, which can be computed at the final stage of the procedure and are presented in Fig. 23. The obtained results also provide the reactive power dispatch assigned to $\mathrm{PV}$ (a $\pm 20 \%$ reactive power capability with respect to active power is assumed in PV inverters) and VSI units associated with energy storage devices, as is depicted in Figs. 24 and 25.

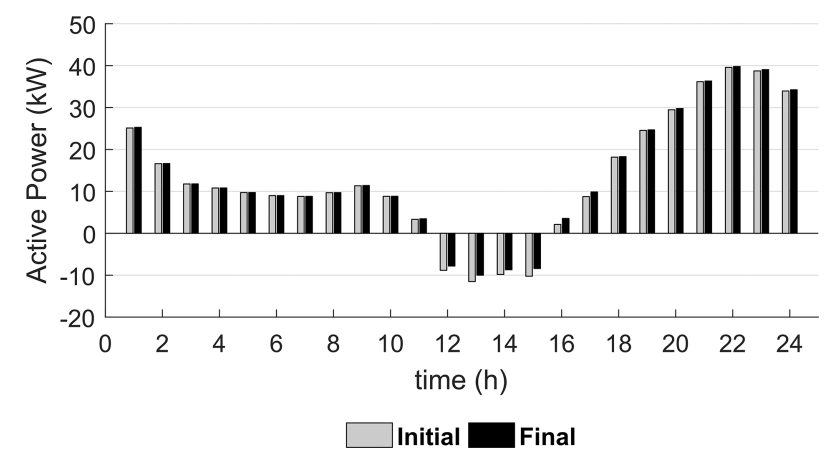

Fig. 20 VSII initial versus final active power scheduling

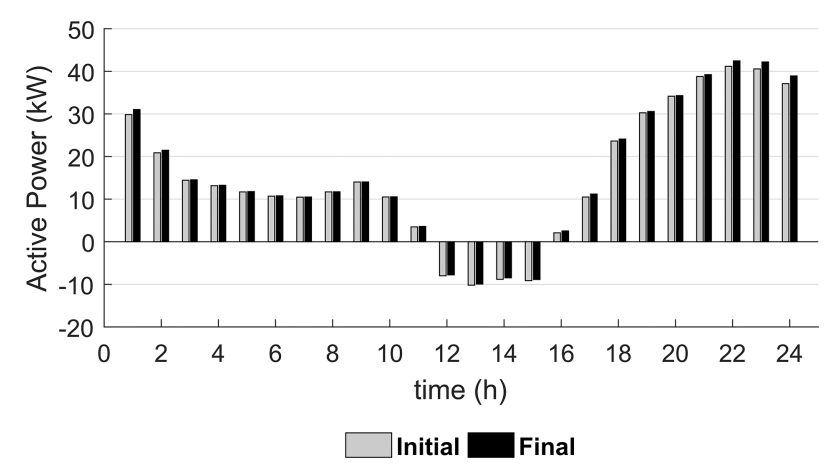

Fig. 21 VSI4 initial versus final active power scheduling

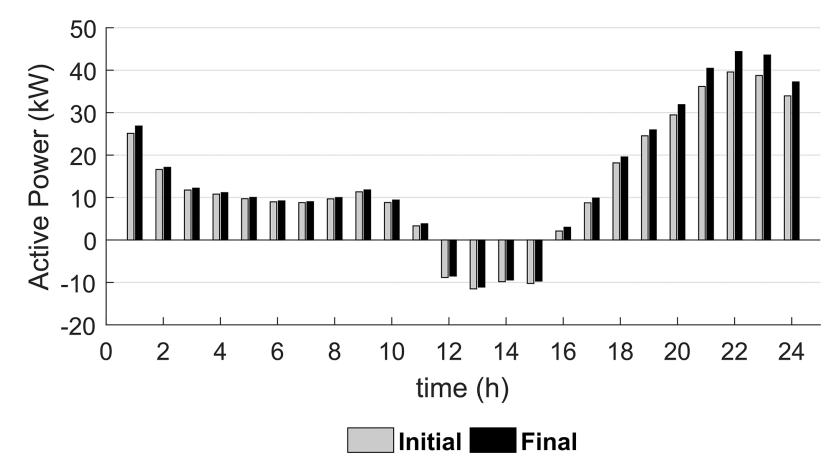

Fig. 22 VSI5 initial versus final active power scheduling

6.3.2 Active power losses analysis: As depicted in Fig. 23, the active power losses among all MGs are very similar in the first 11 h. After that, the differences become more pronounced, especially in two time windows. During peak solar time, the MG1 operation generated the highest active power losses. In contrast, the period of higher power consumption corresponds to higher losses in the other MG, especially in MG2. The MV active losses can be disregarded in comparison with the losses of the LV grids, as shown in Fig. 23. Besides, the total active losses never become higher than $7 \mathrm{~kW}$.

In order to address the robustness of the proposed approach, a fully centralised algorithm for the energy scheduling and voltage/var control problem of the considered MGC was executed at the central MGC controller level for the autonomous operation window of the system (it consists of a centralised multi-temporal active power dispatch, as presented in Section 4.3, defining setpoints for the available resources). In comparative terms, the total hourly system losses that were obtained with the proposed methodology and the centralised algorithm are depicted in Fig. 26. These results demonstrate good adherence between the two options, which contributes to the validation of the proposed approach that benefits from the previously discussed advantages. The active power losses obtained from the proposed algorithm were slightly higher than the ones resulting from the centralised approach, with an increase of $4.3 \mathrm{kWh}$ observed in the overall energy losses for the considered time horizon where the MGC is operating autonomously. 


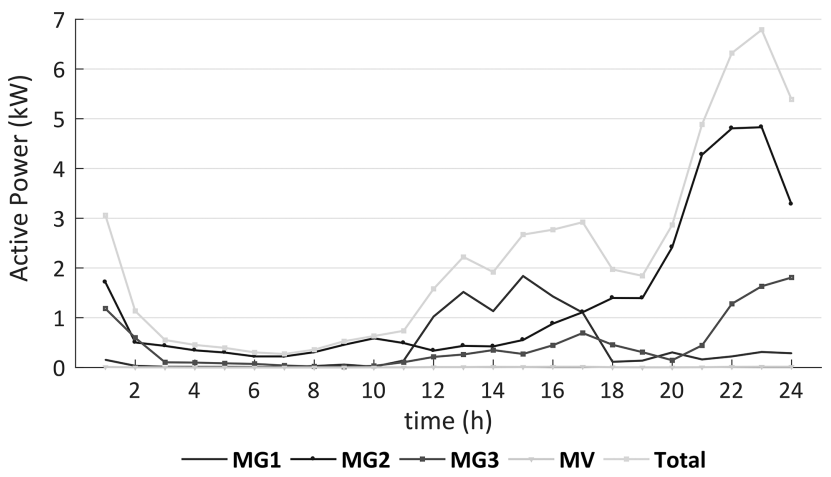

Fig. 23 Active power losses in the cluster of $M G$

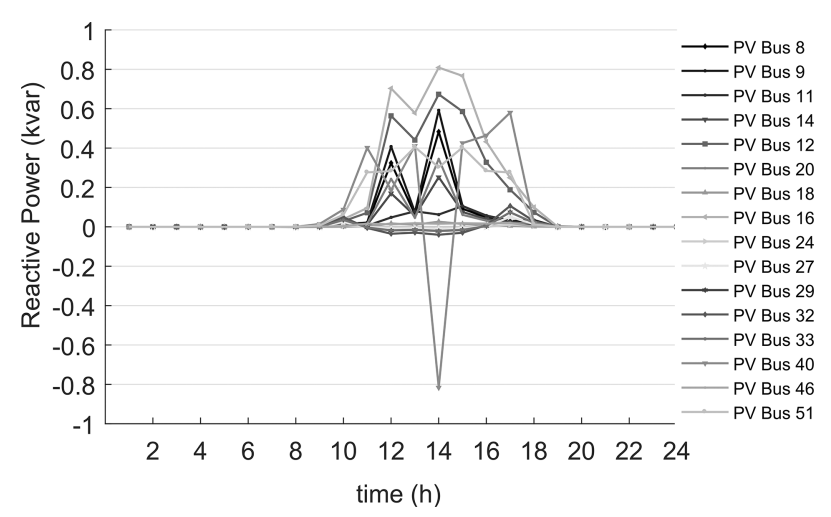

Fig. 24 PV reactive power output schedule

\subsection{Main achievements}

The extensive numerical simulation results that were presented and discussed in detail in Sections 6.1-6.3 provide a clarification/ insight into the capabilities/effectiveness of the proposed approach with respect to the topic developed within this paper. Although this content has significant importance to evidence the relevance of the contribution, it is important to extrapolate these findings to a broader domain. In particular, a higher level reading of the obtained results demonstrates that the theoretical conceptualisation of dividing the energy schedule and volt/var control problem into the MV and LV (MG) stages is valid and works in a robust manner for the considered test system (24 h of MGC autonomous operation). These $24 \mathrm{~h}$ encompassed different scenarios (peak, valley and noon) of production/consumption within the cluster. In addition, the developed tool successfully scheduled the energy within the MMG and addressed the volt/var control problem without compromising the technical operation limits of the grid and the devices connected to it.

Therefore, the main achievement derived from this paper relies on the methodological approach duly validated under extensive numerical simulations regarding the operational management of a MGC during autonomous operation. The envisioned approach fully relies on well-established architectures for the operation of future distribution system under a hierarchical/distributed concept - the MG and MMG systems. In this sense, the proposed approach merits rely on its modularity/adaptability to be incorporated on those architectures.

\section{Conclusion}

A multi-temporal hierarchical algorithm for the energy dispatch and volt/var control problem in MGC operating autonomously was proposed in this study. This approach exploits the MMG concept and its modular architecture by splitting the computational effort and control responsibilities between the CAMC and the MGCC. These operational levels are portrayed in the tool as MV and LV optimisation stages, with the need of coordinating proper information exchange among them in order to allow the practical implementation of the intended procedure of both energy dispatch and volt/var control. Simulation results show that the application of

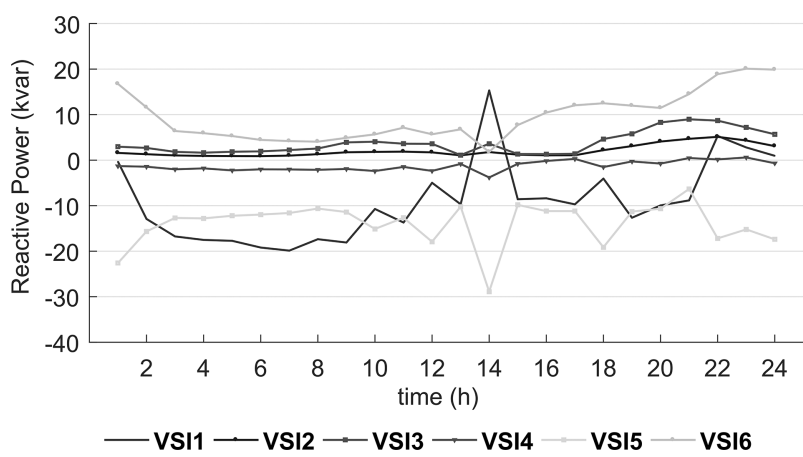

Fig. 25 VSI reactive power output schedule

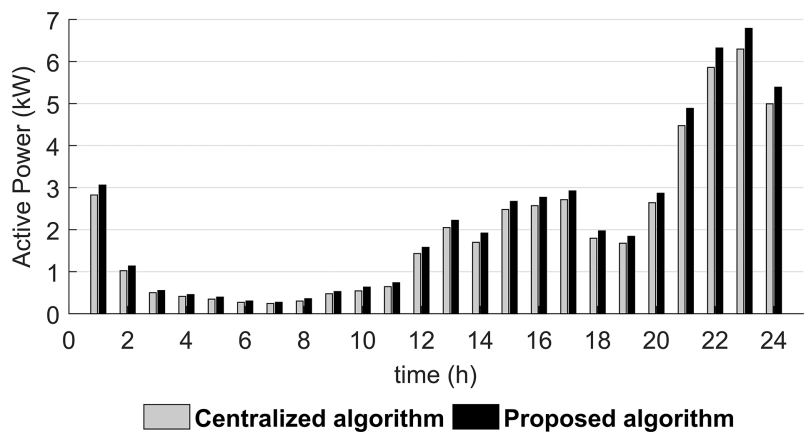

Fig. 26 Losses comparison between algorithms

the conceptual hierarchical optimisation scheme to a MGC autonomously operating during $24 \mathrm{~h}$ was successful and, therefore, valid. Furthermore, the proposed optimisation strategy provided a solution with slightly higher overall active power losses (in this case $4.3 \mathrm{kWh}$ ) when compared to a centrally run optimisation. In summary, the most significant contribution of this work is that it provides an optimisation strategy capable of exploiting the modularity, interoperability and scalability characteristics of the MMG.

This work can be further developed and expanded by the deployment of experimental tests in a real MGC in order to evaluate non-functional requirements of the proposed methodology, as well as the implementation of communication protocols to support the conceptual framework presented. Furthermore and taking into account that MIPS was used in this work, an efficiency comparison between different optimisation algorithms, such as meta-heuristics, to tackle the optimisation problems described in this study can be a subject of future research.

\section{Acknowledgments}

This work was financed by the ERDF - European Regional Development Fund through the Operational Programme for Competitiveness and Internationalization - COMPETE 2020 Programme and by National Funds through the FCT - Fundação para a Ciência e a Tecnologia (Portuguese Foundation for Science and Technology) within project 'ERANETLAC/0005/2014'.

\section{References}

[1] Lopes, J.A.P., Madureira, A.G., Moreira, C.L.: 'A view of microgrids', Wiley Interdiscip. Rev. Energy Environ., 2013, 2, (1), pp. 86-103

[2] Farzin, H., Fotuhi-Firuzabad, M., Moeini-Aghtaie, M.: 'Enhancing power system resilience through hierarchical outage management in multimicrogrids', IEEE Trans. Smart Grid, 2016, 7, (6), pp. 2869-2879

[3] Elizondo, M., Liu, C.-C., Schneider, K., et al.: 'Evaluating the feasibility to use microgrids as a resiliency resource', IEEE Trans. Smart Grid, 2017, 8, (2), pp. 687-696, doi: 10.1109/TSG.2015.2494867

[4] Turitsyn, K., Elmoursi, M.S., Al Hosani, M., et al.: 'Adaptive voltage and frequency control of islanded multi-microgrids', IEEE Trans. Power Syst., 2017, 33, (4), pp. 4454-4465, doi: 10.1109/TPWRS.2017.2780986

[5] Mohammadi, A., Bahrami, S.: 'An overview of future microgrids', Smart Microgrids, 2019, pp. 1-6, doi: 10.1007/978-3-030-02656-1_1 
[6] Lopes, J.A.P., Moreira, C.L., Madureira, A.G.: 'Defining control strategies for microgrids islanded operation', IEEE Trans. Power Syst., 2006, 21, (2), pp. 916-924

[7] Han, H., Liu, Y., Sun, Y., et al.: 'An improved droop control strategy for reactive power sharing in islanded microgrid', IEEE Trans. Power Electron., 2015, 30, (6), pp. 3133-3141

[8] Han, H., Hou, X., Yang, J., et al.: 'Review of power sharing control strategies for islanding operation of AC microgrids', IEEE Trans. Smart Grid, 2016, 7, (1), pp. 200-215

[9] Han, Y., Li, H., Shen, P., et al.: 'Review of active and reactive power sharing strategies in hierarchical controlled microgrids', IEEE Trans. Power Electron., 2017, 32, (3), pp. 2427-2451

[10] Olivares, D.E., Canizares, C.A., Kazerani, M.: 'A centralized energy management system for isolated microgrids', IEEE Trans. Smart Grid, 2014, 5, (4), pp. 1864-1875

[11] Lu, T., Wang, Z., Ai, Q., et al.: 'Interactive model for energy management of clustered microgrids', IEEE Trans. Ind. Appl., 2017, 53, (3), pp. 1739-1750

[12] Bui, V., Hussain, A., Kim, H.: 'A multiagent-based hierarchical energy management strategy for multi-microgrids considering adjustable power and demand response', IEEE Trans. Smart Grid, 2018, 9, (2), pp. 1323-1333

[13] Sandgani, M.R., Sirouspour, S.: 'Coordinated optimal dispatch of energy storage in a network of grid-connected microgrids', IEEE Trans. Sustain. Energy, 2017, 8, (3), pp. 1166-1176

[14] Asimakopoulou, G.E., Dimeas, A.L., Hatziargyriou, N.D.: 'Leader-follower strategies for energy management of multi-microgrids', IEEE Trans. Smart Grid, 2013, 4, (4), pp. 1909-1916

[15] Amini, M.H., Boroojeni, K.G., Dragicevic, T, et al: 'A comprehensive cloud-based real-time simulation framework for oblivious power routing in clusters of DC microgrids'. IEEE 2nd Int. Conf. on Direct Current Microgrids, Nuremberg, Germany, 2017, pp. 270-273

[16] Marnay, C., Chatzivasileiadis, S.: 'Microgrid evolution roadmap'. Int. Symp. on Smart Electric Distribution Systems and Technologies, Vienna, Austria, 2015, pp. 139-144

[17] Madureira, A.G.: 'Coordinated and optimized voltage management of distribution networks of distribution networks with multi-microgrids'. FEUP, 2014

[18] Simpson-Porco, J.W., Shafiee, Q., Dorfler, F., et al.: 'Secondary frequency and voltage control of islanded microgrids via distributed averaging', IEEE Trans. Ind. Electron., 2015, 62, (11), pp. 7025-7038

[19] Castro, M.V., Moreira, C.L.: 'Multi-temporal active power scheduling and voltage/var control in autonomous microgrids' in 'Green energy and networking' (Springer, Guimarães, Portugal, 2019), pp. 193-207

[20] John, T., Ping Lam, S.: 'Voltage and frequency control during microgrid islanding in a multi-area multi-microgrid system', IET Gener. Transm. Distrib., 2017, 11, (6), pp. 1502-1512

[21] Wu, X., Shen, C., Iravani, R.: 'Feasible range and optimal value of the virtual impedance for droop-based control of microgrids', IEEE Trans. Smart Grid, 2017,8 , (3), pp. 1242-1251

[22] Kampezidou, S., Vasios, O., Meliopoulos, S.: 'Multi-microgrid architecture: optimal operation and control'. 2018 North American Power Symp., Fargo, ND, USA, 2019, pp. 1-5

[23] Meirinhos, J.L., Rua, D.E., Carvalho, L.M., et al.: 'Multi-temporal optimal power flow for voltage control in MV networks using distributed energy resources', Electr. Power Syst. Res., 2017, 146, pp. 25-32

[24] Sun, X., Zhang, W.: 'Coordinated optimal voltage control strategy in distribution networks with multi-microgrids'. Asia-Pacific Power Energy Engineering Conf. (APPEEC), Kota Kinabalu, Malaysia, 2018, 2018-Octob, pp. $88-93$

[25] McKay, C.: 'Energy storage networks'. Available at https:// www.energystoragenetworks.com/three-battery-types-work-grid-scaleenergy-storage-systems/, accessed October 2018

[26] Zimmerman, R.D., Murillo-Sánchez, C.E.: 'MATPOWER 6.0 user's manual'. PSerc, 2016, (December), pp. 1-47

\section{Appendix}

The tables and figures present in this section are related to the specific details of the MGC used in the case study. Table 3 exhibits the nominal value of power consumption associated with each bus of the MGC, as well as its base voltage (in $\mathrm{kV}$ ). In Table 4, the branches' electrical characteristics are displayed in S.I. units. Table 5 shows the nominal apparent power and leakage reactance of the transformers. The PV installed active power capacity is detailed in Table 6. Finally, Fig. 10 portrays a single line diagram of the MGC grid as a visual aid and to complement the information of the tables.
Table 3 Active and reactive nominal power demand and base voltage

\begin{tabular}{|c|c|c|c|}
\hline Bus ID & $\begin{array}{l}\text { Active power } \\
\text { demand, kW }\end{array}$ & $\begin{array}{l}\text { Reactive power } \\
\text { demand, kVar }\end{array}$ & Base V, kV \\
\hline 1 & 0.00 & 0.00 & 15 \\
\hline 2 & 0.00 & 0.00 & 15 \\
\hline 3 & 0.00 & 0.00 & 15 \\
\hline 4 & 0.00 & 0.00 & 0.4 \\
\hline 5 & 0.00 & 0.00 & 0.4 \\
\hline 6 & 0.00 & 0.00 & 0.4 \\
\hline 7 & 0.00 & 0.00 & 0.4 \\
\hline 8 & 8.05 & 1.63 & 0.4 \\
\hline 9 & 8.53 & 2.61 & 0.4 \\
\hline 10 & 13.43 & 2.73 & 0.4 \\
\hline 11 & 4.25 & 1.07 & 0.4 \\
\hline 12 & 2.14 & 0.63 & 0.4 \\
\hline 13 & 0.37 & 0.05 & 0.4 \\
\hline 14 & 7.00 & 1.42 & 0.4 \\
\hline 15 & 2.11 & 0.43 & 0.4 \\
\hline 16 & 2.08 & 0.68 & 0.4 \\
\hline 17 & 2.90 & 0.59 & 0.4 \\
\hline 18 & 7.83 & 1.12 & 0.4 \\
\hline 19 & 3.32 & 0.83 & 0.4 \\
\hline 20 & 5.42 & 1.58 & 0.4 \\
\hline 21 & 0.00 & 0.00 & 0.4 \\
\hline 22 & 0.00 & 0.00 & 0.4 \\
\hline 23 & 2.30 & 0.51 & 0.4 \\
\hline 24 & 14.00 & 2.80 & 0.4 \\
\hline 25 & 7.00 & 1.40 & 0.4 \\
\hline 26 & 5.42 & 1.09 & 0.4 \\
\hline 27 & 7.83 & 1.96 & 0.4 \\
\hline 28 & 3.00 & 0.60 & 0.4 \\
\hline 29 & 0.00 & 0.00 & 0.4 \\
\hline 30 & 2.08 & 0.42 & 0.4 \\
\hline 31 & 9.00 & 1.80 & 0.4 \\
\hline 32 & 0.00 & 0.00 & 0.4 \\
\hline 33 & 0.00 & 0.00 & 0.4 \\
\hline 34 & 14.00 & 2.80 & 0.4 \\
\hline 35 & 25.00 & 6.25 & 0.4 \\
\hline 36 & 0.00 & 0.00 & 0.4 \\
\hline 37 & 0.00 & 0.00 & 0.4 \\
\hline 38 & 0.00 & 0.00 & 0.4 \\
\hline 39 & 9.05 & 1.81 & 0.4 \\
\hline 40 & 9.53 & 2.38 & 0.4 \\
\hline 41 & 13.43 & 2.73 & 0.4 \\
\hline 42 & 4.25 & 1.07 & 0.4 \\
\hline 43 & 4.14 & 0.83 & 0.4 \\
\hline 44 & 6.23 & 1.56 & 0.4 \\
\hline 45 & 7.00 & 1.42 & 0.4 \\
\hline 46 & 5.11 & 1.02 & 0.4 \\
\hline 47 & 15.67 & 3.13 & 0.4 \\
\hline 48 & 4.34 & 0.87 & 0.4 \\
\hline 49 & 7.83 & 1.12 & 0.4 \\
\hline 50 & 9.33 & 2.33 & 0.4 \\
\hline 51 & 5.42 & 1.58 & 0.4 \\
\hline
\end{tabular}


Table 4 Branches' resistance, reactance, and susceptance

\begin{tabular}{|c|c|c|c|c|}
\hline From bus & To bus & $R, \Omega$ & $X, \Omega$ & $B, \mathrm{~S}$ \\
\hline 1 & 2 & 0.00095 & 0.00020 & 0.15013 \\
\hline 2 & 3 & 0.00048 & 0.00010 & 0.07506 \\
\hline 4 & 7 & 0.00000 & 0.15708 & 0.00000 \\
\hline 6 & 13 & 0.00000 & 0.15708 & 0.00000 \\
\hline 5 & 11 & 0.00000 & 0.15708 & 0.00000 \\
\hline 7 & 8 & 0.08100 & 0.02932 & 0.00000 \\
\hline 8 & 9 & 0.01600 & 0.00200 & 0.00000 \\
\hline 8 & 10 & 0.03800 & 0.01380 & 0.00000 \\
\hline 10 & 13 & 0.00400 & 0.00099 & 0.00000 \\
\hline 13 & 20 & 0.19900 & 0.02378 & 0.00000 \\
\hline 13 & 19 & 0.02800 & 0.00352 & 0.00000 \\
\hline 13 & 18 & 0.01800 & 0.00190 & 0.00000 \\
\hline 10 & 12 & 0.00900 & 0.00206 & 0.00000 \\
\hline 12 & 16 & 0.00200 & 0.00038 & 0.00000 \\
\hline 12 & 17 & 0.02100 & 0.00502 & 0.00000 \\
\hline 10 & 11 & 0.02100 & 0.00256 & 0.00000 \\
\hline 11 & 15 & 0.02100 & 0.00256 & 0.00000 \\
\hline 11 & 14 & 0.06500 & 0.00493 & 0.00000 \\
\hline 21 & 22 & 0.00000 & 0.15708 & 0.00000 \\
\hline 22 & 23 & 0.08100 & 0.02932 & 0.00000 \\
\hline 23 & 24 & 0.01600 & 0.00200 & 0.00000 \\
\hline 23 & 25 & 0.03800 & 0.01380 & 0.00000 \\
\hline 25 & 26 & 0.00400 & 0.00099 & 0.00000 \\
\hline 26 & 29 & 0.19900 & 0.02378 & 0.00000 \\
\hline 26 & 28 & 0.02800 & 0.00352 & 0.00000 \\
\hline 26 & 27 & 0.01800 & 0.00190 & 0.00000 \\
\hline 25 & 31 & 0.00900 & 0.00206 & 0.00000 \\
\hline 31 & 34 & 0.00200 & 0.00038 & 0.00000 \\
\hline 31 & 35 & 0.02100 & 0.00502 & 0.00000 \\
\hline 25 & 30 & 0.02100 & 0.00256 & 0.00000 \\
\hline 30 & 32 & 0.02100 & 0.00256 & 0.00000 \\
\hline 30 & 33 & 0.06500 & 0.00493 & 0.00000 \\
\hline 36 & 38 & 0.00000 & 0.15708 & 0.00000 \\
\hline 37 & 41 & 0.00000 & 0.15708 & 0.00000 \\
\hline 38 & 39 & 0.08100 & 0.02932 & 0.00000 \\
\hline 39 & 40 & 0.01600 & 0.00200 & 0.00000 \\
\hline 39 & 41 & 0.03800 & 0.01380 & 0.00000 \\
\hline 41 & 44 & 0.00400 & 0.00099 & 0.00000 \\
\hline 44 & 50 & 0.19900 & 0.02378 & 0.00000 \\
\hline 44 & 51 & 0.02800 & 0.00352 & 0.00000 \\
\hline 44 & 49 & 0.01800 & 0.00190 & 0.00000 \\
\hline 41 & 43 & 0.00900 & 0.00206 & 0.00000 \\
\hline 43 & 47 & 0.00200 & 0.00038 & 0.00000 \\
\hline 43 & 48 & 0.02100 & 0.00502 & 0.00000 \\
\hline 41 & 42 & 0.02100 & 0.00256 & 0.00000 \\
\hline 42 & 45 & 0.02100 & 0.00256 & 0.00000 \\
\hline 42 & 46 & 0.06500 & 0.00493 & 0.00000 \\
\hline
\end{tabular}

Table 5 Transformers' leakage reactance and nominal apparent power

\begin{tabular}{lccc}
\hline From bus & To bus & XI, \% & S, kVA \\
\hline 1 & 7 & 6 & 250 \\
2 & 22 & 6 & 250 \\
3 & 38 & 6 & 250 \\
\hline
\end{tabular}

Table 6 PV nominal active power generation

\begin{tabular}{lc}
\hline Bus ID & Active power generation, $\mathrm{kW}$ \\
\hline 8 & 12.50 \\
9 & 12.50 \\
11 & 14.40 \\
14 & 10.50 \\
12 & 35.20 \\
20 & 7.50 \\
18 & 5.20 \\
16 & 40.50 \\
24 & 1.20 \\
27 & 5.20 \\
29 & 7.50 \\
32 & 10.50 \\
33 & 7.50 \\
40 & 32.00 \\
46 & 5.20 \\
51 & 29.00 \\
\hline
\end{tabular}

